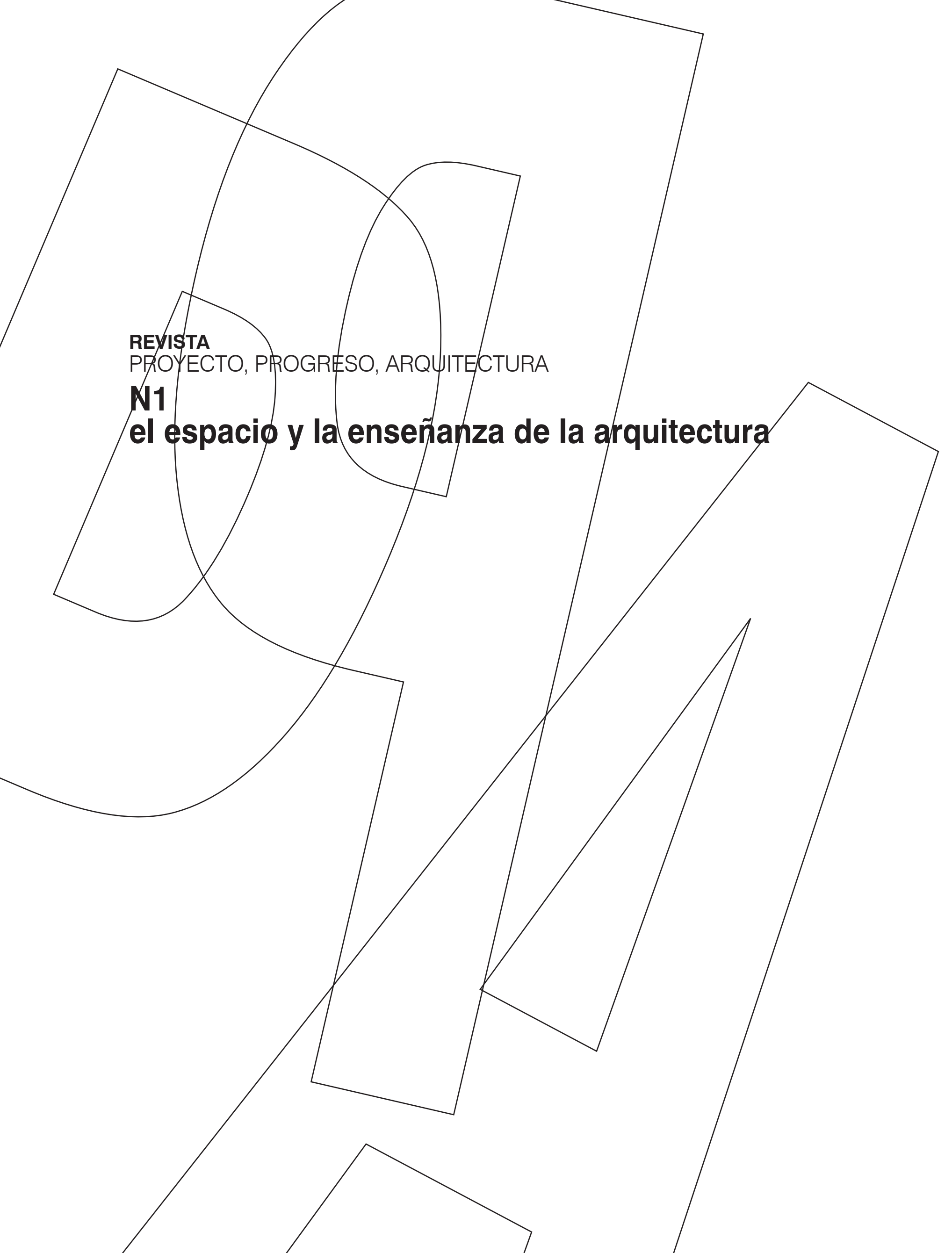




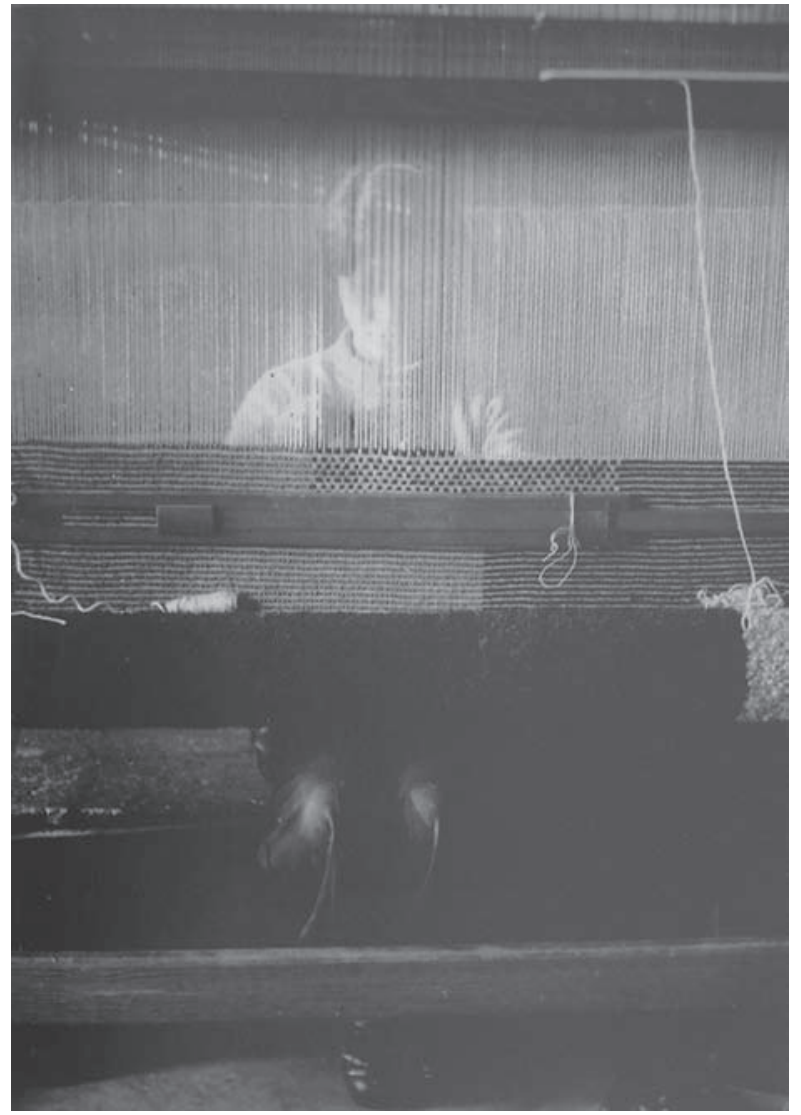

RESUMEN La elaboración actual de un plan de estudios es un proceso de una gran complejidad. Para afrontarlo, son necesarias una serie de estrategias que se encuentran vinculadas al orden. Este artículo explica la manera en que se han aplicado los distintos conceptos de orden, no sólo en la estructuración formal del plan de estudios, sino también en los procesos didácticos, el desarrollo temporal del aprendizaje y la elaboración de los contenidos de las materias en la E.T.S. de Arquitectura del Vallés.

PALABRAS CLAVE Crespo, Mària, plan de estudios arquitectura, orden aprendizaje, ETSA Vallès.

SUMMARY The present production of a curriculum is a process of great complexity. In order to confront it, a series of strategies are necessary that are linked to organization. This article explains the way in which the different concepts of organization have been applied, not only in the formal structuring of the curriculum, but also in the didactic processes, the temporal development of the learning and the production of the contents of the subjects in the Vallès Higher Technical School of Architecture (ETSAV).

KEY WORDS Crespo, Mària, architecture curriculum, learning organization, ETSA Vallès.

Persona de contacto / Corresponding author: isabel.crespo@upc.edu 


\section{EL ORDEN NECESARIO. ALGUNAS CUESTIONES RELACIONADAS CON EL PLAN DE ESTUDIOS DE LA ETSAV}

ESSENTIAL ORGANIZATION: SOME QUESTIONS RELATED TO THE CURRICULUM OF ETSAV

Isabel Crespo Cabillo, Magda Mària i Serrano

\section{CUESTIONES PREVIAS: DESBROZAR}

n el momento de plantearse el diseño de un

$E$ plan de estudios se abre todo un mundo de posibilidades. Entran nuevamente en juego un importante número de parámetros: desde las materias y asignaturas a los contenidos y créditos de las mismas; desde la metodología docente a los sistemas de evaluación; desde la secuencia de las enseñanzas a su extensión temporal. Algunos de estos parámetros se encuentran vigentes y otros, generalmente los más numerosos, se añaden de nuevo. Al mismo tiempo, vuelven a aparecer sobre la mesa todas aquellas cuestiones relacionadas con la experiencia acumulada: la tradición, la inercia de las estructuras existentes, las categorías del conocimiento, la metodología ejercida por generaciones de docentes... Y, cómo no, también resuenan todo tipo de opiniones y críticas, lanzadas desde los más variopintos sectores de la sociedad.

El diseño de los planes de estudio actuales, encaminado a la construcción de un Espacio Europeo de Educación Superior, ha sufrido un largo periplo que se ha prolongado durante demasiado tiempo. Este hecho, unido a diversos cambios políticos, ha contribuido a enturbiar todavía más el ya de por sí complejo proceso de adaptación europea ${ }^{1}$. Después de todo el ruido producido, después de tantos años discutiendo demasiado, escuchando mucho, leyendo notas y manifiestos, adaptando leyes, normas y regulaciones a veces contradictorias, analizando planes piloto y propuestas varias, se han garabateado muchos borradores hasta llenar de negro el papel. La tarea ha consistido, pues, en desbrozar.

Apartar el grano de la paja, desenredar el entuerto formado por todos los datos recogidos, distinguir lo esencial de lo anecdótico, con el objetivo de que un centro inquieto como el nuestro, la Escuela Técnica Superior de Arquitectura del Vallés, reconocido por su agilidad en la generación de cambios y ajustes en los sistemas docentes, no pierda aquello más sustancial de sus enseñanzas. Y, al mismo tiempo, no desperdicie la oportunidad de revisar los procesos y los contenidos vigentes, de manera que la reelaboración del plan de estudios contribuya a la formación de los arquitectos que en un futuro inmediato han de poner sus conocimientos y experiencias al servicio de la sociedad.

\section{LA ESTRATEGIA DEL ORDEN}

Una vez desbrozado el panorama, una vez distinguidos las componentes principales de los secundarios, una vez fijadas las cuestiones ideológicas que no se deben perder, pero también aquellas que se han de revisar o incorporar, es necesario el establecimiento de una estrategia para elaborar el documento.

Organizar todo el conjunto de variables identificadas, y todas aquellas que pueden aparecer de nuevo, implica impulsar el manejo de un sinfín de combinaciones. Como ocurre con la confección de un paño o con la elaboración de un cesto, los componentes pueden entrelazarse de infinitas maneras, tejiendo objetos de formas y texturas muy distintas. La dificultad, pero también el desafío, estriba en el modo en que los elementos finalmente se engarzan para formalizar, de manera concreta, el mejor de los tejidos o cestos posibles que, además, ofrezca la flexibilidad suficiente para adaptarse a circunstancias distintas y arropar contenidos cambiantes, sin por ello perder su identidad (figura 1).

Ante este reto, los parámetros atribuidos al orden resultan imprescindibles para establecer un patrón sobre el que construir, de entre las múltiples combinaciones posibles, el modelo que ofrezca la mejor formación de 
acuerdo con las necesidades del mundo actual y que, a su vez, permita reubicar con facilidad las indudables variaciones y los necesarios ajustes que vayan surgiendo a partir de las nuevas coyunturas.

Entendemos que hay orden cuando una pluralidad de miembros, elementos o partes está dominada por una ley, un sentido o una unidad. La determinada relación recíproca entre las partes, y de éstas con el todo, se encuentra siempre, siguiendo el concepto clásico de orden, vinculada a una idea. Sin embargo el orden, según la definición moderna, no está tan relacionado con un principio jerárquico o ideológico sino que reside en las cosas mismas en cuanto son conocidas, y en las relaciones referenciales que se establecen entre ellas ${ }^{2}$. Así, tanto la disposición de una pluralidad de elementos en virtud de una organización dirigida a alcanzar una determinada idea, como la estrategia vehiculada hacia el conocimiento verdadero de la naturaleza de las cosas y a la articulación de las relaciones que de ello se deriva, conviven de manera habitual en el concepto de orden.

Las cualidades de disposición, jerarquía y conexión vinculadas al orden, aplicadas a un plan de estudios, permiten conocer, distinguir, priorizar, distribuir, organizar y relacionar de manera abierta o cerrada los distintos parámetros que entran en juego. Los factores ideológicos y de conocimiento vinculados al orden, sirven para dotar de contenidos al plan de estudios y se encuentran indisolublemente unidos, en cada una de las escuelas españolas, a una determinada manera de entender la arquitectura. En la Escuela de Arquitectura del Vallès, este determinado enfoque arquitectónico se sustenta desde hace años en la vinculación de la ideación creativa a su concreta materialización, haciendo hincapié en las cualidades habitables de los espacios, en las relaciones que éstos establecen con la sociedad y la cultura, en el respeto por la historia y el paisaje y en el uso responsable de los recursos.

Pero esta manera de entender la arquitectura que establecen y desarrollan algunas escuelas es también, en sí misma, una estrategia de orden. Elegir, priorizar y relacionar; vincular las partes con el todo según unos principios jerárquicos e ideológicos; decidir en función del conocimiento profundo de las cosas; establecer vínculos entre entidades distintas; organizar, idear y conocer no son, en definitiva, patrimonio de la renovación de un plan de estudios, sino que se encuentran íntimamente vinculados a la disciplina arquitectónica. Le Corbusier afirma que la arquitectura es organización ${ }^{3}$. Mies apela directamente al orden como sistema imprescindible a la hora de trabajar en un proyecto ${ }^{4}$. Y Kahn se refiere al orden de los problemas y al orden de la naturaleza de los espacios, de los que nace el proyecto. ${ }^{5}$ Por tanto, el proceso arquitectónico, como el que atañe a la configuración de un plan de estudios, se fundamenta, entre otras cosas, en todos aquellos procedimientos que, provengan del clasicismo o de la modernidad, se encuentran vinculados al orden.

Por ello, además de los parámetros indisolublemente unidos a la arquitectura que contemplan todos aquellos matices tangibles e intangibles que, más allá de la razón, forman parte de la creación arquitectónica. Creemos que la presencia intrínseca del orden en el ejercicio disciplinar, constituye un referente ideal para que el desarrollo de su aprendizaje se encuentre estructurado de manera análoga.

\section{EL ORDEN DE LAS ETAPAS: ORDEN DIRIGIDO Y ORDEN AUTOGESTIONADO}

Un elemento que parece clave para una universidad de calidad, y que la estructura que se propone en los acuerdos de Bolonia persigue, es el establecimiento de dos etapas académicas. La primera afianza en buena medida la troncalidad específica de la disciplina. La segunda debe servir para desarrollar la capacidad del estudiante de dirigir su propio aprendizaje.

El orden dirigido que rige la primera parte de los estudios, se organiza a lo largo de 6 cuatrimestres. En este periodo, marcadamente instrumental y de carácter obligatorio, se organizan temporalmente los contenidos de las materias y asignaturas de manera que todos ellos coincidan en unos objetivos cuatrimestrales comunes. El alumno va aprendiendo de manera gradual y relacionada, los conocimientos necesarios para afrontar la creciente complejidad del resto de la carrera. 
A diferencia del orden dirigido de la primera etapa, la segunda etapa, plenamente universitaria, puede ser insertada en una estructura curricular flexible, utilizando la opcionalidad en la matrícula de las asignaturas obligatorias y ofertando un amplio abanico de optativas de calidad. La principal ventaja es la capacidad de autogestión de las asignaturas, tan reclamada desde el estudiantado y tan necesaria para dirigir el propio perfil formativo.

La libre configuración curricular es una estructura organizativa relativamente fácil de incorporar en la segunda parte de la carrera. Habitualmente, en esta última etapa, las promociones se desdibujan y, especialmente en arquitectura, es difícil identificar qué estudiantes pertenecen a uno u otro curso. La mayoría de ellos están matriculados en asignaturas de diferentes cuatrimestres, no por haber fracasado, sino porque la duración de la carrera hace que deban compatibilizar los estudios con su vida laboral o personal. Por lo tanto, reconocer este aparente desbarajuste y hacer creíble que el orden de matrícula de las asignaturas de los dos últimos años pueda construirlo el propio estudiante, no transgrede lo establecido, sino que contribuye a formar una madurez deseable en una persona de esa edad. Aquí el orden se flexibiliza, se hace adaptable al que aprende, como un material maleable pendiente de esculpir al servicio de objetivos de aprendizaje coherentes en sí mismos.

En este entreno inmejorable con vistas al futuro, en el que se inicia el proceso autorregulado de seguir aprendiendo a lo largo de toda la vida profesional, se abre la puerta a que el futuro arquitecto adquiera un determinado perfil, en función de las materias que ha cursado. En la actualidad, cada vez más voces autorizadas insisten en afirmar que un licenciado no puede saber de todo con la intensidad que reclama el ejercicio profesional. El recién titulado no puede ser un especialista de nada. El arquitecto, de hecho, no lo es, porque su cometido es, sobre todo, tomar decisiones en la coordinación de los trabajos que hacen posible la materialización del proyecto. ${ }^{6}$ En este cometido ha de demostrar la capacidad de entender qué papel juega cada especialista para poder coordinar sus funciones, siempre desde el punto de mira de la arquitectura.

Tenemos, por tanto, varias componentes que corroboran el orden flexible de los últimos cursos: la conveniencia de ofrecer un cierto perfil formativo que singularice al titulado, unas promociones desdibujadas, y la posibilidad de hacer efectivo un auto aprendizaje propio y característico de un segundo ciclo universitario. La apuesta del plan de estudios de la Escuela de Arquitectura del Vallés pretende dar respuesta a todo ello.

En este orden flexible juegan un papel fundamental los talleres verticales. Esta organización de funcionamiento se implantó en la Escuela del Vallés el año 2004, y consiste en la oferta cuatrimestral de 6 o 7 talleres multidisciplinares de arquitectura impartidos por profesores de proyectos juntamente con profesores de otras materias.

2. FERRATER MORA, José, Diccionario de Filosofía. Ed. Ariel, Barcelona, 1988. vol. III, pp. 2646-2649.

3. "Arquitectura es organización. Tú eres un organizador, no un estilista de tablero de diseño". LE CORBUSIER, "If I had to teach you architecture". Focus, 1. Summer, 1938. Versión traducida al italiano en: Casabella, 766. Maggio 2008, pp. 5-7.

4. "Cada decisión lleva a un tipo de orden particular (...) Debemos poner el acento en el orden derivado de un principio orgánico, como medio para establecer una feliz relación entre las partes y entre éstas con el todo. (...) El largo camino que nos transporta del material al trabajo creativo a través de la función tiene solamente un único fin: traer el orden a la desesperante confusión de nuestro tiempo". MIES van der ROHE, Ludwig, "Inaugural Address". Conferencia pronunciada como Director del Departamento de Arquitectura del Armour Institut de Chicago, 1938. Versión traducida al italiano en: "Ludwig Mies van der Rohe sull'insegnamento dell'architettura". Casabella, 767. Giugno 2008, pp. 3-5.

5. Kahn explica el proceso de diseño en base a tres etapas: Naturaleza del Espacio, Orden y Diseño. También se refiere al Orden como un concepto implícito en la creación formal: "Las formas con las que se experimenta vienen de un conocimiento más cercano de la naturaleza y de nuestra constante búsqueda de orden. Los hábitos que en el diseño llevan a ocultar la presencia de la estructura no tienen cabida en este orden implícito. Dichos hábitos retardan el desarrollo de la arquitectura como arte”. Ver: TYNG, Anne G.: "Louis I. Kahn's Order in the Creative Process” En LATOUR, Alessandra (ed.), Louis I. Kahn: I'uomo, il maestro. Ed. Kappa. Roma, 1986, pp. 277-290. LATOUR, Alessandra (ed.), Louis I. Kahn, escritos, conferencias y entrevistas. El Croquis ed. Madrid, 2003, pp. 45-46.

6. SIZA, Alvaro, "Sulla pedagogia". Casabella, 770, octubre 2008, pp. 3-5. 
Los estudiantes pueden escoger, desde el séptimo cuatrimestre, uno de esos talleres. En el octavo tienen la opción de elegir otro, y así hasta el décimo. En éste último empiezan a trabajar en lo que debe acabar siendo su proyecto final de carrera. La consecuencia es que en cada taller se encuentran estudiantes de cuatro niveles, más los estudiantes extranjeros de intercambio. En estos talleres los temas abarcan registros diversos vinculados a temas de actualidad. Si imaginamos que, simultáneamente a un taller de restauración, un estudiante cursa una asignatura obligatoria de patología de la construcción, una optativa de gestión del patrimonio o de construcción tradicional y, al mismo tiempo, realiza unas prácticas en el departamento de patrimonio de una administración local, está construyendo un perfil propio muy determinado y le está dando a su carrera un sesgo muy claro que en ese cuatrimestre se dirige hacia la restauración.

La implicación del estudiante en la confección de este perfil es evidente y viene a completar el mecanismo de convalidación de asignaturas cursadas en el extranjero con los intercambios interuniversitarios. Las asignaturas que se convalidan tras un intercambio no necesariamente se ubican en el mismo año, si atendemos a los contenidos u objetivos formativos equivalentes. Por tanto, el currículum se "desordena". Visto así, el intercambio es una opción más, que se suma a las ofrecidas por el propio centro. Resulta evidente que, por ello, la armonización europea ha de pasar necesariamente por la armonización de las guías docentes. En ellas, cualquier estudiante de Europa ha de poder entender con claridad qué conocimientos se imparten y cómo se aprenden en cada una de las asignaturas presentes en los planes de estudio de las escuelas europeas.

\section{EL ORDEN DE CONTENIDOS: ORDEN INSTRUMENTAL Y ORDEN COMPLEJO \\ El proceso de aprendizaje del propio alumno dentro de este doble marco de funcionamiento y organización de las etapas, sigue también el argumento a la vez jerárqui- $\mathrm{co}$, relacional e ideológico que se encuentra implícito en el concepto de orden. Frente a planes de estudio que con- templan una transmisión de conocimientos basada en la}

resolución de problemas y tipologías concretas, la estructura del aprendizaje, con un natural incremento temporal de la complejidad, se erige en protagonista de la formación arquitectónica. No importa tanto el qué se aprende sino el cómo se alcanza el conocimiento de manera progresiva y coordinada. No importa tanto el contenido concreto de cada asignatura, sino las conexiones estratégicas que se establecen entre ellas dentro de un mismo curso y, a su vez, las articulaciones funcionales y significativas que enlazan unos cursos con otros. La estructura que ampara estos procesos, como si se tratara de un organismo vivo, designa un conjunto de materias autónomas que se ven obligadas a complementarse compartiendo unos objetivos pedagógicos comunes. En cada curso todas las asignaturas están relacionadas entre ellas, a la vez que su organización responde a una ordenación vinculada con la totalidad de los estudios.

En este sentido, se han buscado unas palabras-clave para cada cuatrimestre, a las que todo el conjunto de asignaturas se refiere, que definen los objetivos formativos comunes y resumen la dirección de su didáctica. Se expresa así la voluntad de acentuar ante todo los procesos que siguen los alumnos para ir adquiriendo los conocimientos a lo largo del transcurso de la carrera, y el orden establecido para ello desde todas las materias.

Los dos primeros cuatrimestres, encabezados por los términos 'contexto, referentes y representación', tienen una clara vocación instrumental, con un progresivo y estudiado acercamiento a los temas que conciernen a la arquitectura. Los alumnos han de adquirir las capacidades básicas que se requieren para comprender y registrar el entorno inmediato, así como conocer los referentes culturales necesarios de la disciplina arquitectónica. Además de los necesarios conocimientos teóricos, las materias proponen el desarrollo práctico de ejercicios bien mesurados, con una direccionalidad clara, y con unos objetivos didácticos precisos.

El tercer y cuarto cuatrimestres, que llevan por título 'sistema y construcción', incorporan a los conocimientos adquiridos en los dos primeros cursos el aprendizaje de mecanismos estratégicos para la construcción y materialización de las formas y para la obtención de espacios 
habitables. El sistema constructivo como táctica de proyecto, pero también el método como habilidad formal y de conocimiento, se erigen en protagonistas de dos cursos en los que la materialidad de la arquitectura y sus condiciones de habitabilidad se encuentran en el centro del discurso.

El quinto y sexto cuatrimestres, siguiendo las palabrasclave que los definen, 'programa, uso y organización', enfocan los temas arquitectónicos desde la planificación, ordenación y distribución de los espacios habitables. Integrando los conocimientos instrumentales y las habilidades tácticas adquiridas en los cuatro primeros cursos, el desarrollo de los trabajos incide en la estructuración de los espacios a partir de programas y funciones, dando respuestas unitarias que pongan en relación las partes con el todo, y relacionando las soluciones con las condiciones del entorno.

Una vez adquiridas, durante el primer ciclo de la carrera, las habilidades básicas para afrontar, desde estrategias de aprendizaje progresivo, problemas arquitectónicos concretos, los cuatro últimos cuatrimestres, titulados 'síntesis, intensificación y programa complejo', exploran temas y contenidos más avanzados, y plantean el conocimiento de la arquitectura desde toda su complejidad. Los temas, susceptibles de estar vinculados a asignaturas optativas de soporte, contemplan las soluciones arquitectónicas desde la investigación experimental y abordan cuestiones sociales, culturales y medioambientales vinculadas a la actualidad y al futuro ${ }^{7}$. Los contenidos restan abiertos a cambios, en función de las circunstancias contextuales del momento. El grado de madurez exigido a los alumnos durante este ciclo en el que han de cursar cuatro cuatrimestres, se incrementa con la oferta de diseñar su propia trayectoria curricular, tal como se ha explicado en el apartado anterior.

En este segundo ciclo, coincidiendo plenamente con los principios establecidos por el EEES, el trabajo que llevan a cabo los alumnos en los Talleres adquiere un papel relevante. En estos espacios de colaboración, la estrategia didáctica, presente en los Planes de Estudio de la Escuela desde 1993, está basada en un enfoque de la arquitectura que relaciona íntimamente el proyecto creativo con su concreta materialización. Esta manera de abordar la ideación arquitectónica, fundamentada en casos reales y no simulados, conlleva los necesarios componentes de interdisciplinariedad y la presencia en el aula de interlocutores docentes de diversas materias, en ejercicio constante de coordinación. Los alumnos enriquecen sus ideas con el diálogo con profesores de especialidades diferentes, que aportan su visión de los problemas desde ángulos distintos. La interrogación, el conflicto y la puesta en cuestión de los temas de proyecto relacionados siempre con su aplicación real, ejercita a enriquecer un espíritu crítico y a entender la arquitectura con toda su complejidad.

\section{EL ORDEN FUTURO}

En paralelo a la elaboración de un plan de estudios se abren una serie de cuestiones que, más allá de las razones organizativas, estructurales, pedagógicas, metodológicas y de contenidos, vinculan la formación universitaria a temas que se encuentran inmersos en la actualidad más candente. Los estudios de arquitectura han de comprometerse también con los paradigmas del presente: el medio ambiente, la habitabilidad, la responsabilidad social, el respeto por las preexistencias, la incorporación de la historia, o la relación con el lugar, entre otros.

Pero de los tiempos presentes asoma una realidad compleja, inmersa en un período de transición, a caballo entre un largo ciclo literario y matérico, protagonizado por la cultura transmitida a través de los libros y por el aprendizaje directo de las cosas, y un ciclo que se avecina interminable, predominantemente virtual, donde los medios informáticos están asumiendo un fuerte protagonismo en la transmisión de la información y en las relaciones interpersonales. Una época en la que conviven los entornos naturales y urbanos de proximidad con los que siempre 
ha trabajado la arquitectura, con el entorno telemático que tiene su recinto en las redes del ciberespacio.

¿Cómo conjugar estas dos realidades en las aulas? ¿Cómo sobrellevar la invasión de una cultura virtual en el aprendizaje de una profesión íntimamente vinculada a la realidad material y a la experiencia humanística? ¿Cómo mantener el necesario equilibrio entre novedad y tradición, entre virtualidad y materialidad, entre espectáculo y oficio?

Es evidente que nuestros estudiantes son personas del presente, que manejan como nadie la información, que se encuentran sumergidos en viajes y en relaciones virtuales y que vienen a clase armados de todo tipo de prótesis artificiales que los conectan al ciberespacio. Pero muchas veces, esta acuciante cantidad de medios a su alcance, los transforma en seres pasivos, impacientes y con una relación quimérica con el contexto inmediato. Alumnos no entrenados a proceder de manera reflexiva, carentes de las dosis de realidad necesarias y poco propensos a la receptividad, paciencia y persistencia que requiere una arquitectura que aspira a ser de calidad.

¿Cómo hacer, pues, compatible el aprendizaje y la enseñanza de una disciplina que requiere el tiempo lento, la sedimentación paulatina de las ideas, la reflexión, la agudeza de los cinco sentidos o el espíritu refinado, en un contexto en el que domina la inmediatez, la impaciencia y la cultura visual?

Este nuevo escenario, todavía desquiciado y con evidentes signos de bipolaridad, necesita también de un largo periodo donde los ciclos se ajusten, donde la sedimentación de cada una de sus cualidades encuentre su justo lugar. Pero sobre todo, requiere un nuevo modelo de orden, seguramente distinto al orden de los clásicos y al vigente orden de los modernos. Un orden que tenga la capacidad de enlazar nuevos estímulos con viejas certezas, de conjugar la acuciante virtualidad con todos aquellos aspectos de la realidad más tangible y humana. ¿Será el aprendizaje de la arquitectura el que pueda dar la clave a la hora de definir este orden futuro? 
Isabel Crespo Cabillo (Barcelona, 1961), arquitecta (1989), doctora arquitecta (2005) por la Universitat Politècnica de Catalunya. Profesora colaboradora doctora de Geometría Descriptiva de la Escuela Técnica Superior de Arquitectura del Vallés (UPC). Miembro del grupo CAIRAT (Centre d'Aplicacions Informàtiques per la Representació de l'Arquitectura i el Territori). Investigadora de la implicación de la geometría en la arquitectura. Editora de la revista Las razones del dibujo técnico, ha publicado en la revista Disegnare $n^{\circ} 35$. Actualmente es Subdirectora-Jefe de Estudios de la ETSAV. Magda Mària i Serrano (Barcelona, 1961), arquitecta (1987), doctora arquitecta (1995) por la Universitat Politècnica de Catalunya. Profesora agregada de Proyectos Arquitectónicos de la Escuela Técnica Superior de Arquitectura del Vallés (UPC), ha sido profesora y miembro del equipo de dirección de la Escuela de Arquitectura La Salle-Barcelona (URL, 1999-2006). Miembro del grupo de investigación HABITAR. Ha publicado los libros Renaixement i arquitectura religiosa y Forma-Pensamiento y artículos en las revistas Tectónica $n^{0} 20$ y Arquitectura Ibérica $n^{0} 32$. Actualmente es Subdirectora de Plan de Estudios de la ETSAV. 